\title{
Slitted leading edge profiles for the reduction of turbulence-aerofoil interaction noise
}

\author{
P. Chaitanya* and P. Joseph ${ }^{\dagger}$ \\ University of Southampton, SO17 1BJ Southampton, UK
}

(Dated: April 6, 2018)

\begin{abstract}
Aerofoils operating in a turbulent flow are an efficient source of noise radiation by scattering vorticity into sound at the leading edge. Much work has now been undertaken demonstrating the effectiveness by which serrations, or undulations, introduced onto the leading edge can substantially reduce broadband leading edge interaction noise. However, all of this work is focused on sinusoidal leading edge serration profiles. In this paper, an alternative profile is proposed that is capable of providing significantly greater noise reductions than the maximum noise reductions that can be achieved by single-wavelength serrations of the same peak-to-root amplitude. In its most general realization, the leading edge serration profiles simply comprise a sawtooth or single wavelength serration, for which every root has a single narrow slit. This simple geometry, upon interaction with a turbulent flow, produces compact source regions at either ends of the slit, which then destructively interfere, leading to considerably less efficient noise radiation than conventional single-wavelength geometries. The paper will demonstrate experimentally that even slits by themselves can provide greater levels of noise reduction than conventional profiles of the same peak-to-root amplitude.
\end{abstract}

PACS numbers: PACS: 43.28.Ra

\footnotetext{
*mail2pcc@gmail.com

†pfj@isvr.soton.ac.uk
} 


\section{INTRODUCTION}

Aerofoils operating in a turbulent flow are an efficient source of noise radiation by scattering vorticity into sound at the leading edge. This noise generation mechanism is common in many industrial examples such as in the interaction between rotor wake turbulence and the leading edge of the downstream Outlet Guide Vanes (OGV) of a turbofan aero engine. Wind turbines are another important unwanted environmental noise source where the interaction of large-scale atmospheric turbulence interacting with the rotating aerofoil blades is believed to the one of the dominant noise source, particularly at very low frequencies.

In recent years there has been considerable interest in the use of leading edge (LE) serrations aimed at reducing interaction noise [1-11]. However, all of these papers are focused on single-wavelength serrated leading edge profiles. The noise reduction mechanism of these profile have been investigated in detail by $[4,8-10]$ and essentially involves a reduction in the source strength along the serrated leading edge through interference as well as a reduction in the source strength arising from a reduced mean flow velocity component normal to the edge. The result of these mechanisms is that the resultant source distribution is concentrated at the serration roots whose source strength (pressure difference per unit edge length) is roughly equal to that of the straight edge $[9,10]$. The fundamental upper limit on sound power reductions that can be achieved using single wavelength serrations has been identified by [10]. In this paper it was shown that maximum noise reductions occur at the optimum wavelength $\lambda_{0}$, which is roughly four times the transverse integral length scale $\Lambda$ of the incoming turbulent flow. At this optimum wavelength the reduction in sound power level at any frequency $f$ is given by $\Delta \mathrm{PWL}(f)=10 \log _{10}(f h / U)+10$, where $h$ is the serration amplitude and $U$ is the flow velocity.

Recently, [11] demonstrate the influence of turbulence anisotropy on the performance of sinusoidal leading edge serrations. They demonstrated numerically that small variations in the turbulence length scales in the chordwise and spanwise direction can produce significant changes in the spectral content of the noise sources at the peak and root regions. They identified two key ratios that affects noise reduction on single wavelength serrations, one is between the chordwise length scale and the amplitude of the serrations, and second is the ratio between the spanwise length scale and the serration wavelength. However, the influence of anisotropy is beyond the scope of the current work and can be considered in 
further refinements of the technique.

An alternative leading edge profiles were investigated by [12]. One of the profiles in this work considered the sum of two single wavelengths of roughly the same amplitude. This simple profile was shown to provide superior noise reductions to the single wavelength serrations of the same overall amplitude. Simply summing the two wavelengths has the desired effect of producing two dominant compact sources at adjacent roots that are separated in both streamwise and spanwise directions. This geometry was shown to provide additional levels of noise reduction compared to single-wavelength serrations due to destructive interference between these adjacent compact root sources. By virtue of the time taken for eddies to convect between the streamwise distance between adjacent roots, these root-sources radiate out of phase leading to less efficient far field radiation. However, their performance was found to be fundamentally limited by the partial coherence between adjacent sources due to their spanwise separation distance.

In this paper, we propose a leading edge geometry for reducing leading edge interaction noise that significantly exceeds the noise reduction performance (by up to $11 \mathrm{~dB}$ ) of both single and double-wavelength serrations. In the most general form it comprises a sawtooth profile in which narrow slits with typical widths of just a few millimetres are introduced at the root positions. The introduction of these slits, upon interaction with turbulent flow, produces two compact acoustic source regions at either ends of the slit. However, since these sources are aligned in the streamwise direction and the turbulent flow convects along the slit as a frozen pattern, they are highly coherent and their level of destructive interference considerably greater than that of the double wavelength serration. As in the case of the double wavelength serrations, these sources will radiate out of the phase by virtue of the time taken for eddies to convect along the length of the narrow slit. A frequency therefore exists at which these two coherent source regions radiate $180^{\circ}$ out of phase. Providing the source strengths at this frequency are arranged to be equal through appropriate choice of slit width, therefore, perfect cancellation of the sound field can in principle be achieved at this frequency. We will show below that noise reductions of almost $18 \mathrm{~dB}$ in radiated sound power can be achieved under laboratory conditions using this control principle at a flow speed of $40 \mathrm{~m} / \mathrm{s}$, with a small reduction in performance being observed at higher flow speeds. 


\section{SLITTED-ROOT LEADING EDGE SERRATIONS}

\section{A. Geometry}

The Slitted-root leading edge design investigated in this paper involves introducing narrow slits at the serration roots, as sketched in figure 1. In this paper we demonstrate that, for very narrow slits, the noise reduction is almost identical at low frequencies, and better at high frequencies, than that of single-wavelength or sawtooth serration profiles of the same peak-to-root amplitude. However, introducing narrow slits clearly requires much less modification to the aerofoil leading edge as shown in Figure 2 showing a comparison of the two leading edge geometries drawn to scale. However, the main innovation of the new leading edge design is that a new noise reduction mechanism is introduced once the slit width is increased. In this case, we hypothesize that the source strengths at either ends of the slit become comparable and interfere destructively, leading to much greater levels of noise reduction compared to conventional profiles of the same overall amplitude.

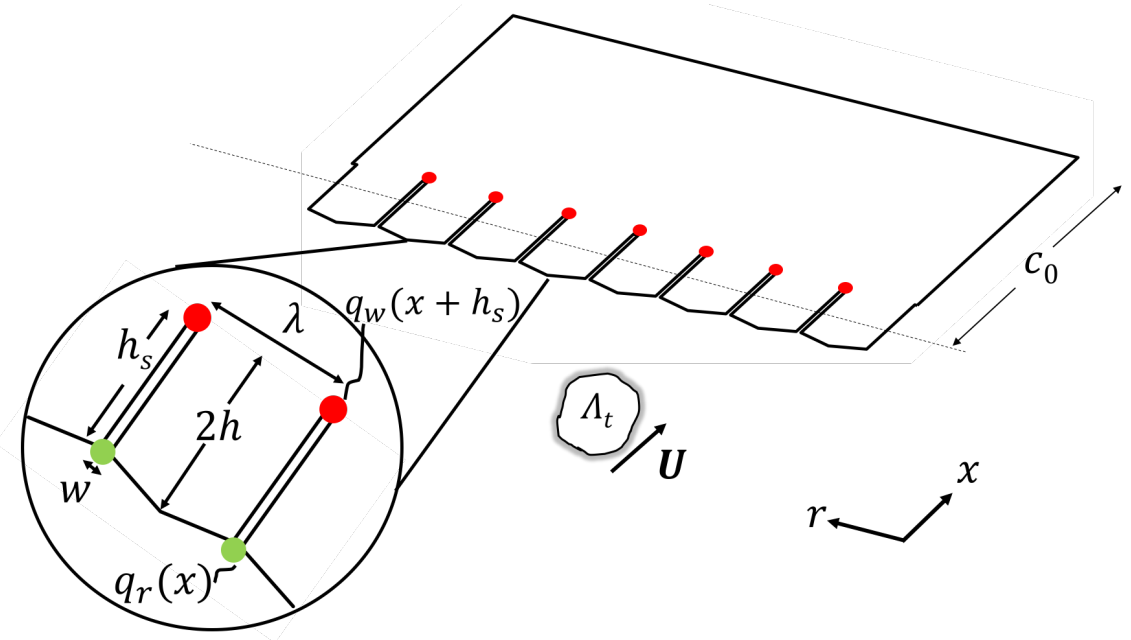

FIG. 1. A sketch of the slitted-root leading edge serration.

The principal dimensions of the slitted root profile are sketched in figure 2. For sufficiently large slit widths $w$ (greater than $0.5 \mathrm{~mm}$ for the geometries under consideration), the noise reduction spectra presented in Section $\mathrm{V}$ exhibit a frequency of maximum noise reduction. This suggests that the dominant sources (indicated as red and green dots) are located at either ends of the slit separated in the streamwise direction by close to the slit height $h_{s}$. The overall distance between the peak and the end of the slit is $2 h$, with the pattern repeating 
with periodicity (wavelength) $\lambda$.

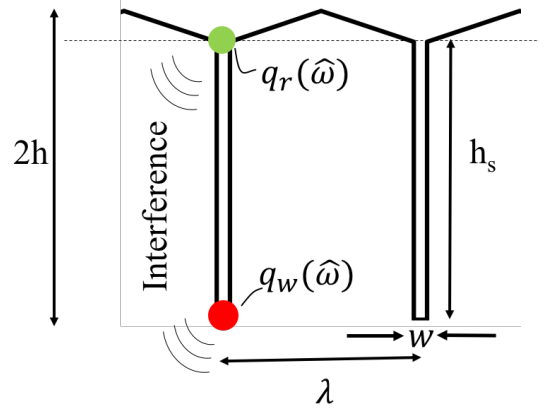

Slitted-root serrations

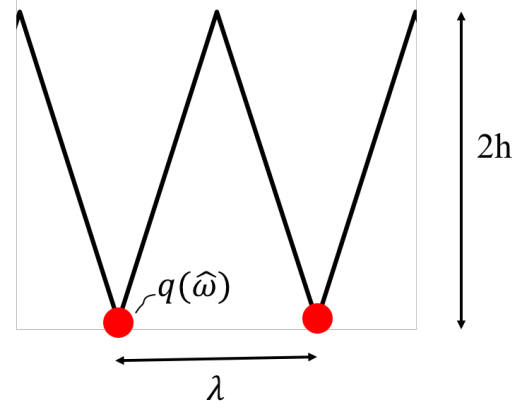

Sawtooth serrations

FIG. 2. A comparison of slitted-root and sawtooth serration. C compared to conventional sawtooth leading edge serrations.

\section{B. A simple analytical model to predict noise reductions}

Turbulent flow interacting with the profile sketched in figure 1 will induce variations in the pressure-difference (between pressure and suction sides) over the aerofoil. The largest pressure-differences principally occur along the leading edge whose strength and phase variation mostly determine the level of noise reduction in the far field. Previous work on singlewavelength profiles indicate that the dominant sources are located at the root region [9-11]. The reason for this behaviour has been investigated in [13]. They showed that the root of the serrated leading edge is the dominant noise source due to the presence of a secondary horseshoe-like vortex system generated by the serrated leading edge, which alters the upstream velocity field, thereby enhancing the source strength at the serration root. The noise reduction spectra shown below in section $\mathrm{V}$ suggests that dominant compact sources are also present in the slitted root profile but with localized sources located at either ends of the slit, as indicated by the green and red dots in figure 1.

Consider two compact sources located at the streamwise locations $x_{1}$ and $x_{1}+h_{s}$ with source strengths $q_{r}\left(x_{1}, \omega\right)$ and $q_{w}\left(x_{1}+h_{s}, \omega\right)$, as shown in figure 1 . However, these source strengths have been shown on single wavelength serration to be solely a function of the non- 
dimensional frequency $\hat{\omega}=\omega h / U$, where $h$ is the overall serration height as demonstrated by [10]. In the case of the slitted root, the source strengths $q_{r}$ and $q_{w}$ are now complicated functions of the slit geometry $q_{r}=q_{r}\left(\omega, h_{s}, h, w, \lambda\right)$ and $q_{w}=q_{w}\left(\omega, h_{s}, h, w\right)$. The path length differences between $q_{r}$ and $q_{w}$ to a far-field observer can be neglected since they are generally much closer together compared to an acoustic wavelength. The total radiated pressure is therefore proportional to the sum of source strengths with relative phase difference included,

$$
p(\omega) \propto q_{r}\left(x_{1}, \omega\right)+q_{w}\left(x_{1}+h_{s}, \omega\right) e^{-i \omega h_{s} / U} .
$$

In this paper we make the assumption that the incoming turbulence is frozen, i.e., $v(\mathbf{x}, t)=v(\mathbf{x}-\mathbf{U} t)$, where $v$ is the velocity component normal to the aerofoil and $\mathbf{x}=(r, x)$ is any position in the plane of the flat plate. Further making the assumption that the turbulence is homogeneous, the space-time correlation function $R=E\left[v\left(x_{1}, t_{1}\right) v\left(x_{1}+h_{s}, t_{2}\right)\right]$ for the velocity $v$ between the two stream-wise positions $x_{1}$ and $x_{1}+h_{s}$ is of the form

$$
R\left(x_{1}, x_{1}+h_{s}, t_{1}, t_{2}\right)=\hat{R}\left(h_{s}-\left(t_{1}-t_{2}\right) U\right) .
$$

where $\hat{R}\left(h_{s}-\left(t_{1}-t_{2}\right) U\right)$ is the spatial correlation function in the reference frame moving with the flow. A direct consequence of this frozen turbulence assumption is that the sources $q_{r}$ and $q_{w}$ at either ends of the narrow slit must be perfectly coherent since the turbulent eddies that excite them simply convect with the mean flow along the slit height $h_{s}$ as a frozen pattern of turbulence. The radiated sound power $W(\omega)$ is proportional to mean square pressure,

$$
W(\omega) \propto \overline{p^{2}}(\omega) \propto \mathbf{E}\left[p^{*}(\omega) p(\omega)\right] .
$$

Substituting (1) into (3), the radiated sound power becomes,

$$
W(\omega) \propto\left[\overline{q_{r}^{2}}(\omega)+\overline{q_{w}^{2}}(\omega)+2 \overline{q_{r}(\omega) q_{w}^{*}(\omega)} \cos \left(\omega h_{s} / U\right)\right] .
$$

where $\overline{q_{r}^{2}}(\omega)$ and $\overline{q_{w}^{2}}(\omega)$ are the mean square source strengths at the frequency $\omega$ at the two locations either ends of the slit and $\overline{q_{r}(\omega) q_{w}^{*}(\omega)}$ represents the cross spectra between the two sources. The advantage of the slitted-root design is that these sources are highly coherent and hence the cross spectrum may be replaced by the product of the rms source strengths, 


$$
\overline{q_{r}(\omega) q_{w}^{*}(\omega)}=\sqrt{\overline{q_{r}^{2}}(\omega) \overline{q_{w}^{2}}(\omega) .}
$$

The sound power radiation $W(\omega)$ from the slitted root serration may now be compared to the baseline un-slitted sawtooth profile of the same overall amplitude $W_{s}(\omega)$, which we assume is due to a single compact source at the root of the serration $q(\omega)([10])$ as sketched in figure 2. The additional sound power level reduction compared to the sawtooth baseline case may therefore be written as,

$\Delta P W L_{A}(\omega)=10 \log _{10}\left(\frac{W_{s}(\omega)}{W(\omega)}\right)=10 \log _{10}\left(\frac{\overline{q^{2}}(\omega)}{\overline{q_{r}^{2}}(\omega)+\overline{q_{w}^{2}}(\omega)+2 \sqrt{\overline{q_{r}^{2}}(\omega) \overline{q_{w}^{2}}(\omega)} \cos \left(\omega h_{s} / U\right)}\right)$.

Overall sound power reductions provided by these slitted-root profiles therefore comprise the sum of two contributions. The first arises from the reduction in source strength along the oblique leading edge, which we shall denote as $\Delta \mathrm{PWL}_{S}$. The second arises solely from interference between the two coherent sources $q_{r}$ and $q_{w}$ at either ends of the slit, which we shall denote as $\Delta \mathrm{PWL}_{A}$. The overall sound power reduction may therefore be written as,

$$
\Delta \mathrm{PWL}(\omega)=\Delta \mathrm{PWL}_{S}(\omega)+\Delta \mathrm{PWL}_{A}(\omega) .
$$

The additional noise reductions due solely to inference effects may therefore be obtained by subtracting the sound power level spectra due to a slitted-root serration from that due to the un-slitted-sawtooth serration of the same peak-to-root amplitude, which may be estimated from $\Delta P W L=10 \log _{10}(f h / U)+10$. Equation (6) for the additional noise reduction spectra makes clear that it is completely governed by two source strength ratios. One, $r(\omega)$, quantifies the relative strengths between the two sources at either ends of the slit,

$$
r^{2}(\omega)=\frac{\overline{q_{w}^{2}}(\omega)}{\overline{q_{r}^{2}}(\omega)} .
$$

The second, $s(\omega)$, quantifies the relative strengths between the source at the root location of the un-slitted profile and the sum of source strengths at either ends of the slit, 


$$
s^{2}(\omega)=\frac{\overline{q^{2}}(\omega)}{\left(\sqrt{\overline{q_{r}^{2}}(\omega)}+\sqrt{\overline{q_{w}^{2}}(\omega)}\right)^{2}} .
$$

The final expression for the additional sound power level reduction in terms of the ratio of source strengths defined in Equations (8) and (9) is given by,

$$
\Delta P W L_{A}(\omega)=10 \log _{10}\left(\frac{s^{2}(\omega)}{1+\frac{2 r(\omega)}{(1+r(\omega))^{2}}\left(\cos \left(\pi \omega / \omega_{0}\right)-1\right)}\right)
$$

where,

$$
\omega_{0}=\pi U / h_{s} .
$$

Equation (10) suggests that additional noise reductions obtained from the slitted root serration compared to the un-slitted profile are due to two factors. The first is due to a modification in the source strengths between the un-slitted $q^{2}(\omega)$ and the slitted profiles $\left(\sqrt{\overline{q_{r}^{2}}(\omega)}+\sqrt{\overline{q_{w}^{2}}(\omega)}\right)^{2}$ as accounted for by the ratio $s(\omega)$. The second factor is due to interference between the two source strengths at either ends of slit whose effect is quantified by the denominator in Equation (10). This term goes to zero, and hence perfect noise cancellation is predicted, when the source strengths at the ends of the slit at the frequency $\omega_{0}$ are equal, i.e., $r\left(\omega_{0}\right)=1$, and radiate exactly $180^{\circ}$ out of phase. Here, we refer to $\omega_{0}$ given by Equation (11) as the tuned frequency of the slitted-root profile.

\section{Limiting cases}

As discussed above, we are concerned with the two limiting cases of slitted-root geometry. One occurs for very small slit width for which the source strength $q_{w}(\omega)$ at the downstream end of the slit tends to zero and hence, $r(\omega) \rightarrow 0$. The other is when the slitted-root parameters are chosen so that the source strengths at either ends of the slit are equal and hence $r(\omega) \rightarrow 1$. We now consider each case in turn. 


\section{Slit width tends to zero}

As the slit width tends to zero the source strength at the end of the slit may be neglected at all frequencies and hence $r(\omega) \rightarrow 0$, Equation (10) becomes,

$$
\Delta P W L_{A}(\omega)=20 \log _{10}(s(\omega))
$$

In this limiting case the sound power reduction is therefore determined solely by $s(\omega)$ the ratio between the source strengths at the root of the baseline sawtooth to that of the total source strengths of the slitted-root. An unexpected finding of this work is that, in this limiting case, at frequencies below the tuned frequency, noise reductions are found to be almost identical to those of a conventional sawtooth serration of the same peak-to root distance $2 h$. The ratio of source strengths between the slitted and un-slitted profiles must therefore be equal in the low frequency limit, i.e, $s(\omega) \rightarrow 1$ for $\omega / \omega_{0} \rightarrow 0$. For very narrow slits, $q_{w}(\omega)$ must be negligible and hence,

$$
\overline{q_{w}^{2}(\omega)} \rightarrow \overline{q^{2}(\omega)}, \quad\left(\omega / \omega_{0} \rightarrow 0, r \rightarrow 0\right)
$$

Very narrow slits therefore provide near identical noise reductions to the conventional sawtooth (and single wavelength) serration profiles of the same amplitude. This finding has potentially significant benefits for the design of low noise aerofoils since introducing narrow slits is a much smaller modification to the aerofoil than conventional sawtooth serrations of the same amplitude.

\section{Optimum slit geometry}

The second limiting case of interest here is when the source strengths at either ends of the slit are exactly equal, which we assume is independent of frequency, i.e., $r(\omega)=1$, which can be obtained through optimal choice of slitted root geometry. In this case, the noise reductions from Equation (10) are given by,

$$
\Delta P W L_{A}(\omega)=20 \log _{10}\left(s(\omega) \sec \left(\pi \omega / 2 \omega_{0}\right)\right)
$$

Noise reductions are now solely controlled by the frequency $\omega$ in relation to the tuned 
frequency $\omega_{0}$. Thus, by simply introducing narrow slits onto a leading edge profile, significantly enhanced noise reductions can be obtained compared to un-slitted profiles of the same amplitude. In the next section, the experimental setup is described which allows the proposed noise reduction mechanisms to be validated experimentally. Subsequent sections will present levels of noise reduction substantially higher (by up to 11dB) than can be achieved with conventional profiles of the same amplitude.

\section{EXPERIMENTAL SETUP AND INSTRUMENTATION}

\section{A. Flat plates leading edge serrations}

For economy and ease of manufacture, a parametric study into the effect of $h_{s}$ and $w$ on noise reductions was performed on flat plates situated within a turbulent flow. A slitted-root design arising from this flat plate study was then applied to a 3-D aerofoil of 5\% thickness, which we show in Section VIII has an almost identical noise reduction spectra to the flat plate case thereby justifying the use of flat plates in this parametric investigation.

The flat plate with a mean chord $\left(c_{0}\right)$ of $150 \mathrm{~mm}$ and span of $450 \mathrm{~mm}$ was constructed by joining together two $1 \mathrm{~mm}$ thick metallic sheets to allow serrated flat plate inserts 2 $\mathrm{mm}$ thick to be inserted between them. All corners were rounded and the trailing edge sharpened to eliminate vortex shedding noise. Further details of this flat plate construction can be found in $[6]$.

A total of 18 flat plate slitted serrations of varying slit width, height and wavelength were investigated to explore the sensitivity on noise reductions to these parameter variations, which are listed in table I. Note that in this paper values of $\lambda$ and $h$ are presented as quantities normalized on the mean chord $c_{0}$ although there is no evidence to suggest that this is a meaningful normalization parameter in determining noise reductions.

\section{B. Open-jet test facility and instrumentation}

Far-field noise measurements were carried out at the Institute of Sound and Vibration Research's open-jet wind tunnel facility. The wind tunnel is located within the anechoic chamber, of dimension $8 \mathrm{~m} \times 8 \mathrm{~m} \times 8 \mathrm{~m}$ as shown in figure 3 . The walls are acoustically treated with glass wool wedges and the cut-off frequency is $80 \mathrm{~Hz}$. The nozzle has 


\begin{tabular}{|c|c|c|c|c|}
\hline configuration & $\begin{array}{c}\text { serration } \\
\text { amplitude } \\
\left(h / c_{0}\right)\end{array}$ & $\begin{array}{c}\text { serration } \\
\text { wavelength } \\
\left(\lambda / c_{0}\right)\end{array}$ & slit width $(w / \lambda)$ & $\begin{array}{c}\text { slit amplitude } \\
\left(h_{s} / 2 h\right)\end{array}$ \\
\hline slitted & 0.167 & 0.1 & $\begin{array}{c}0.033,0.067, \\
0.1,0.133, \\
0.167,0.2,0.233\end{array}$ & 0.4 \\
\hline slitted & 0.167 & 0.1 & 0.133 & $\begin{array}{c}0.64,0.7,0.8,0.8,1 \\
0.9,1\end{array}$ \\
\hline sawtooth & $0.1,0.167$ & 0.1 & 0 & 0 \\
\hline sinusoidal & 0.1 & 0.1 & 0 & 0 \\
\hline
\end{tabular}

TABLE I. Leading edge slitted profiles considered in this study

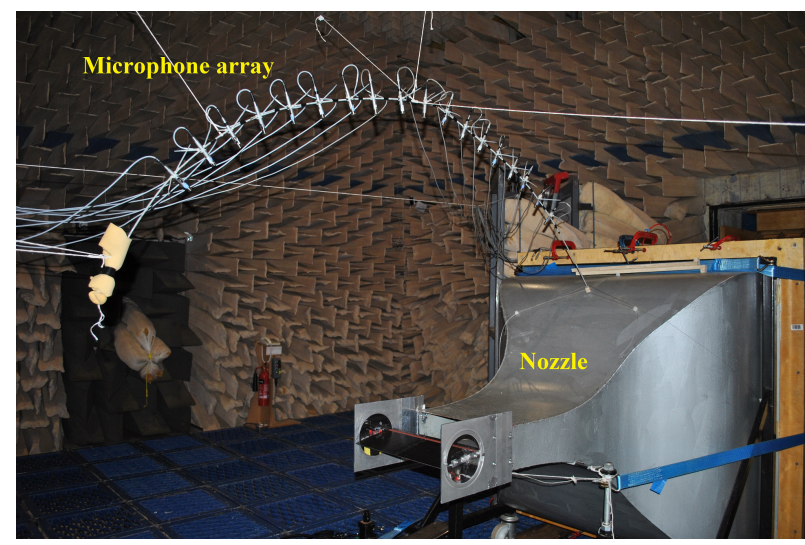

FIG. 3. Photograph of jet nozzle and test setup inside the ISVR anechoic chamber.

dimensions of $150 \mathrm{~mm}$ and $450 \mathrm{~mm}$ and provides a maximum flow speed of $100 \mathrm{~m} / \mathrm{s}$. A detailed description of the wind tunnel, including its characteristics, is presented by [14]. To maintain two-dimensional flow around the flat plate, side plates are mounted to the nozzle exits which will also support the flat plate and aerofoil in the flow. The mean leading edge of the flat plate is located $150 \mathrm{~mm}$ downstream from the nozzle exit.

In order to prevent tonal noise generation due to Tollmien-Schlichting waves convecting in the laminar boundary layer, and to ensure complete consistency between the different cases, the flow near the leading edge of the aerofoil was tripped to force transition to turbulence using a rough band of tape of width $1.25 \mathrm{~cm}$ located $5 \mathrm{cms}$ downstream from the leading edge corresponding to a position on the main body downstream of the serration, on both suction and pressure sides. The tape has roughness of SS 100, corresponding to a surface roughness of $140 \mu m$. 


\section{Far-field noise measurements}

Far-field noise measurements from the flat plate were made using 11, half-inch condenser microphones (B\&K type 4189) located at a constant radial distance of $1.2 \mathrm{~m}$ from the mid span of the flat plate leading edge. These microphones are placed at emission angles of between $40^{\circ}$ and $140^{\circ}$ measured relative to the downstream jet axis. Measurements were carried for $10 \mathrm{~s}$ duration at a sampling frequency of $50 \mathrm{kHz}$, and the noise spectra were calculated with a window size of 1024 data points corresponding to a frequency resolution of $48.83 \mathrm{~Hz}$ and a Bandwidth-Time product (BT) of about 500, which is sufficient to ensure negligible variance in the spectral estimate at this frequency resolution.

The acoustic pressure at the microphone was recorded at the mean flow velocities $(U)$ of 20,40, 60 and $80 \mathrm{~m} / \mathrm{s}$. Noise reductions are presented in terms of the Sound Power Level spectra PWL $(f)$ calculated by integrating the pressure spectra over the polar array of 11 microphones using the procedure described in [6]. Sound power level reductions are determined by subtracting the sound power level spectra due to the serrated flat plate from that due to the baseline straight edge profile.

\section{Turbulence characterization}

A bi-planar rectangular grid of wooden bars of $12 \mathrm{~mm}$ width separated by $34 \mathrm{~mm}$ was used to generate turbulent flow that provides a velocity spectrum that is a close approximation to homogeneous and isotropic turbulence at the aerofoil leading edge. The overall grid dimensions of $630 \times 690 \mathrm{~mm}^{2}$ was located in the contraction section $75 \mathrm{~cm}$ upstream of the nozzle exit. However, we emphasize that the condition of isotropy is not a key requirement for predicting the noise radiation but only that the velocity spectrum at the aerofoil leading edge is known. A comparison of the streamwise velocity spectra measured at $145 \mathrm{~mm}$ from the nozzle exit $\left(S_{u u} / U\right)$ plotted against $f / U$ is compared in figure 4 to the theoretical Liepmann velocity spectrum, where the mean square velocity and integral length scale are chosen to give best fit to the measured data. Close agreement is observed for $2.5 \%$ turbulence intensity and a $7.5 \mathrm{~mm}$ streamwise integral length-scale. The integral length-scale $(\Lambda)$ associated 


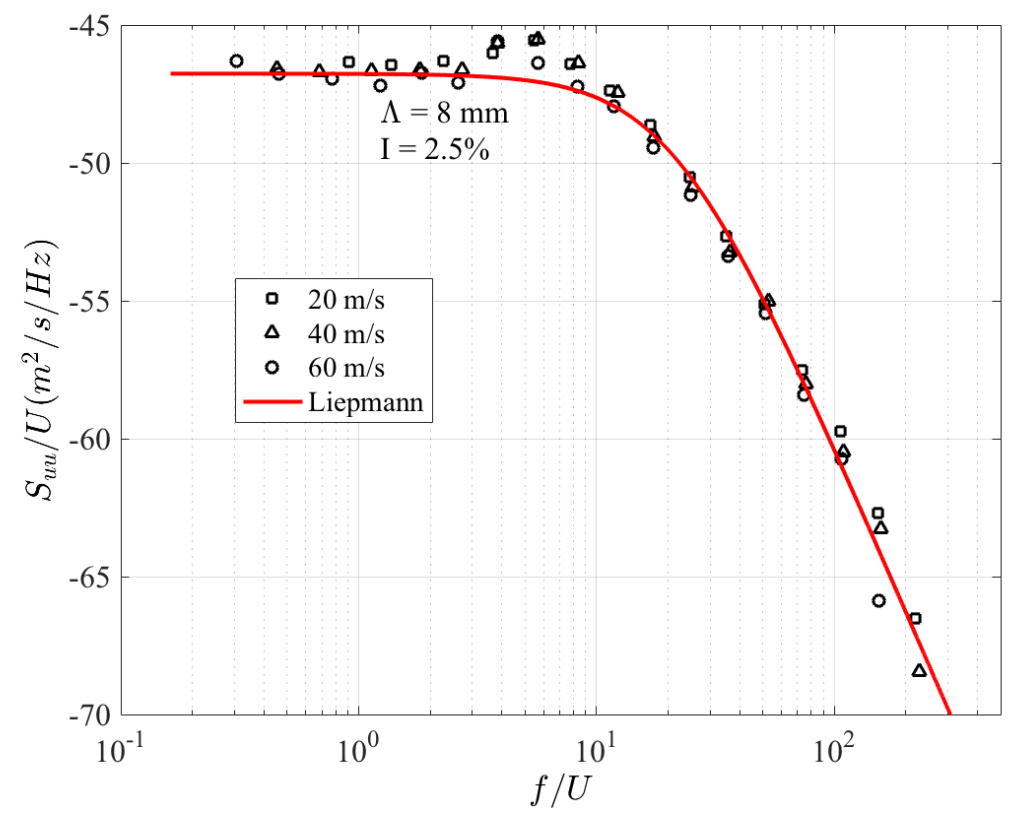

FIG. 4. Comparison between the measured axial velocity spectra and theoretical Liepmann spectra.

with the transverse velocity component (responsible for noise generation on a flat plate) was inferred from one half the streamwise length scale to be $3.75 \mathrm{~mm}$ based on the assumption of isotropy. We emphasise, however, that these turbulence parameters estimates are not required to understand or predict the noise reduction spectra but simply to provide an approximate description of the incoming turbulent flow.

\section{ACOUSTIC PERFORMANCE OF SAWTOOTH SERRATIONS}

Before considering the effects of introducing slits at the serration roots we first consider the noise reduction performance of sawtooth serrations alone without slits. The performance of this un-slitted geometry will form the baseline performance by which the slitted geometries are compared at the same overall amplitude. Nearly all leading edge serration profiles investigated previously were restricted to sinusoidal profiles ([1-11]). We show below that the sawtooth profiles offer marginally better noise reductions at high frequencies but follows roughly the same frequency dependence at the optimum wavelength.

Figure 5 is a comparison between the noise reduction spectra (compared to straight leading edge) plotted against non-dimensional frequency $f h / U$ due to a sawtooth and sinusoidal serration of the same wavelength $\lambda / c_{0}=0.1$ and amplitude $h / c_{0}=0.1$. The wavelengths 


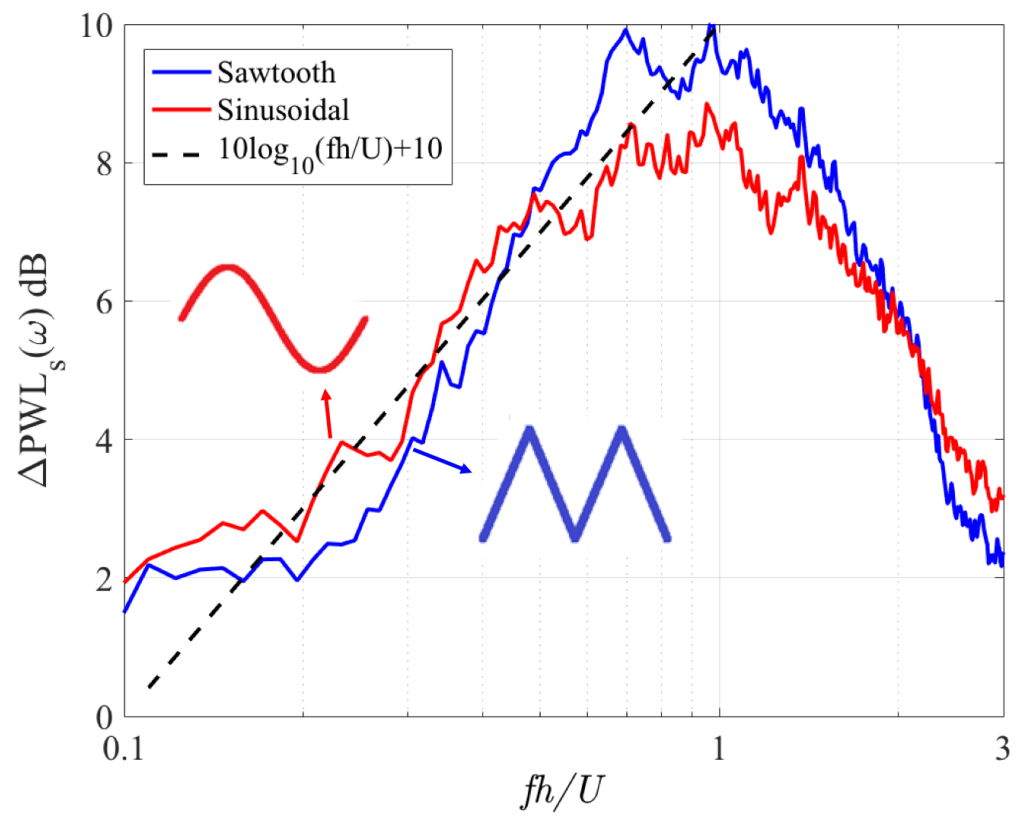

FIG. 5. Comparison of noise reduction spectra (compared to straight leading edge) between sinusoidal and sawtooth leading edge serrations.

are chosen to be 4 times the integral length-scale $\Lambda$ of the incoming turbulence, identified in [10]. This wavelength was shown to be the optimal wavelength for providing maximum noise reductions given by $10 \log _{10}(f h / U)+10$ for single-wavelength profiles, shown in this figure as the dashed line.

Sawtooth serrations can be observed to provide slightly poorer noise reductions $(<1 \mathrm{~dB})$ at frequencies less than about $f h / U=0.5$ compared to the corresponding sinusoidal case, but superior noise reductions at higher frequencies by almost $2 \mathrm{~dB}$. Clearly, therefore, the noise reduction mechanism discussed above is highly sensitive to the details of the root geometry. At frequencies greater than $f h / U=2$, however, both serration profiles become dominated by trailing edge self noise and their performance begin to diminish ([6]).

\section{ACOUSTIC PERFORMANCE OF SLITTED-ROOT SERRATIONS}

\section{A. Typical noise reductions of Slitted-root serrations}

We start by demonstrating the superior noise reduction performance of slitted-root serrations compared to the more conventional un-slitted profiles of the same amplitude. Fig- 
ure 6 shows the sound power reduction spectra plotted against non-dimensional frequency $f h / U$ for a typical slitted-root serration with serration amplitude $h / c_{0}=0.167$, slit width $w / \lambda=0.133$, slit height $h_{s} / 2 h=0.64\left(h_{s} / c_{0}=0.213\right)$ at the four flow speeds of 20,40 , 60 and $80 \mathrm{~m} / \mathrm{s}$. Also shown the sound power reduction spectra for conventional sawtooth serrations of same serration amplitude $h / c_{0}=0.167$ at flow speed of $60 \mathrm{~m} / \mathrm{s}$ along with the line $10 \log _{10}(f h / U)+10$ representing the optimum sound power reduction achievable for a single-wavelength serration at the optimum wavelength.

The noise reduction spectra due to the slitted root serration can be seen to peak at a frequency very close to the tuned frequency $\omega_{0}$ marked on this figure as a vertical arrow. The noise reduction at this peak frequency is substantially higher by about $11 \mathrm{~dB}$ than the corresponding level of the optimum sawtooth geometry of the same amplitude. A second peak in the noise reduction spectra is also apparent at approximately three times the tuned frequency, also marked on this figure. Overall, the slitted-root profile considerably outperforms the single sawtooth profile of the same amplitude. The characteristics of the noise reduction spectra are entirely consistent with the hypothesis of destructive interference between two dominant compact source regions of roughly equal strength at either ends of the slit, as sketched in Figure 1. Almost perfect collapse of the noise reduction spectra at the four different flow speeds are obtained with greatest deviations occurring at the peak frequency, demonstrating that the ratio between the serration characteristic length and the hydrodynamic wavelength $U / f$ is the important parameter in determining the level of noise reductions. Note also that in the low frequency range $f h / U<0.6$ where interference effects are negligible, there is no significant different in noise reductions between the slitted and un-slitted case. Therefore, in the low frequency limit, the source strength ratio $s(\omega)=1$.

In the next section the sensitivity of noise reduction to slit width $w$ is investigated, with particular emphasis on highlighting the different noise reduction mechanisms involved in the limit of very small slit width, $w \rightarrow 0$, and the optimum slit width when $r(\omega) \rightarrow 1$.

\section{B. Influence of $w$ on additional noise reductions}

Figure 7 shows the spectra of noise reductions $\Delta P W L_{A}(\omega)$ additional to the noise reductions achievable with a single-sawtooth serration of the same amplitude and optimum wavelength. The spectra are now plotted against non-dimensional frequency $\omega / \omega_{0}$ to make 


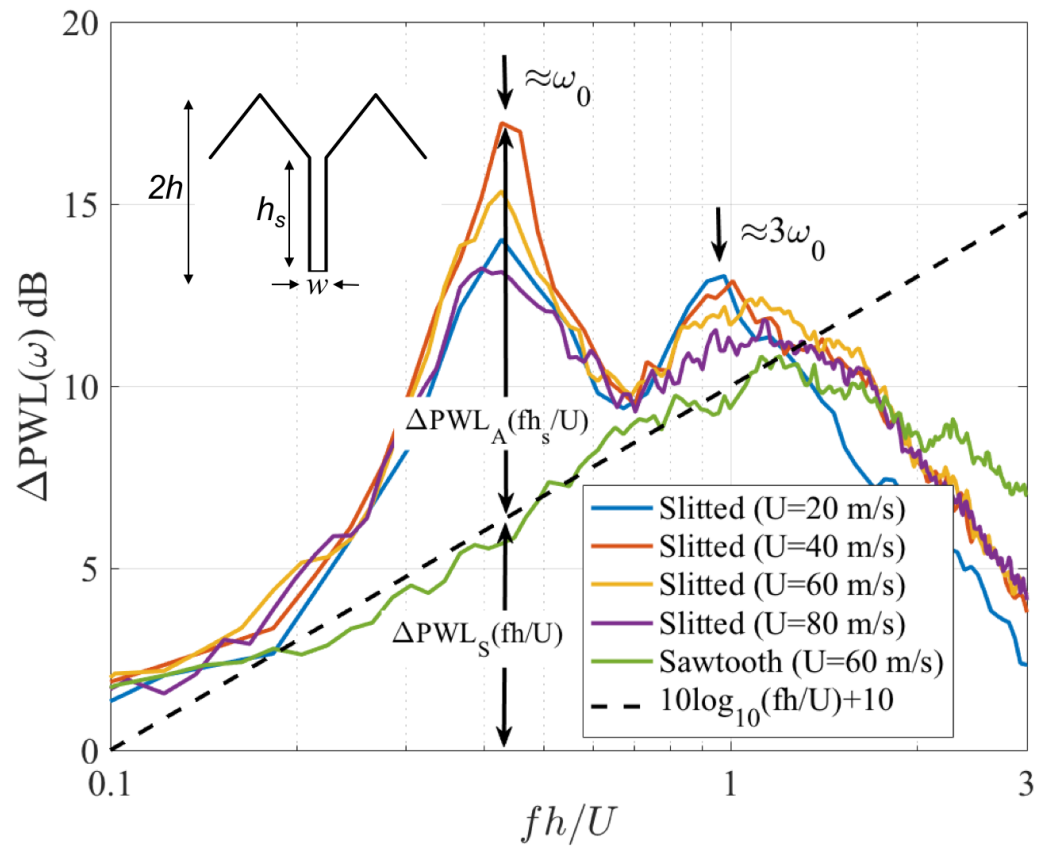

FIG. 6. Influence of jet velocity on noise reductions of slitted-root serrations of slit width $w / \lambda=$ 0.133 , slit amplitude $h_{s} / 2 h=0.64$ and serration amplitude $h / c_{0}=0.167$.

explicit the role of interference in the noise reduction mechanism. The results from 7 different slit widths $w / \lambda$ of $0.033,0.067,0.1,0.133,0.167,0.2$ and 0.233 are presented at a fixed slit height $h_{s} / 2 h=0.4\left(h_{s} / c_{0}=0.133\right)$, serration amplitude $h / c_{0}=0.167$ and flow speed of $60 \mathrm{~m} / \mathrm{s}$. Also shown in this figure are Equations $20 \log _{10} s(\omega)$ and $20 \log _{10}\left(s(\omega) \sec \left(\pi \omega / 2 \omega_{0}\right)\right)$ representing the theoretical additional noise reduction spectra for the two limiting cases of zero slit width and the optimum slit width respectively, where we have used a value of $s(\omega)=1$. Note that $s(\omega)=1$ represents the upper limiting value obtained in the limit of zero slit width. Increasing the slit width from zero has the effect of increasing the root source strength by virtue of the increased edge length and hence reducing $s(\omega)$.

We emphasize that, in general, $s(\omega)$ is not known. However, $s(\omega)=1$ was chosen for consistency with the results of figures 7 and 6 , which both indicate that, for all slit widths under investigation, in the low frequency limit, the slitted and un-slitted serrations provide nearly identical noise reductions, and hence we can infer that $s(\omega) \rightarrow 1$ in this limit.

The results in Figure 7 provide an overview of the advantages of introducing slits at the serration roots whilst maintaining the same overall amplitude.

The noise reduction spectra in this figure may be summarized as follows: 


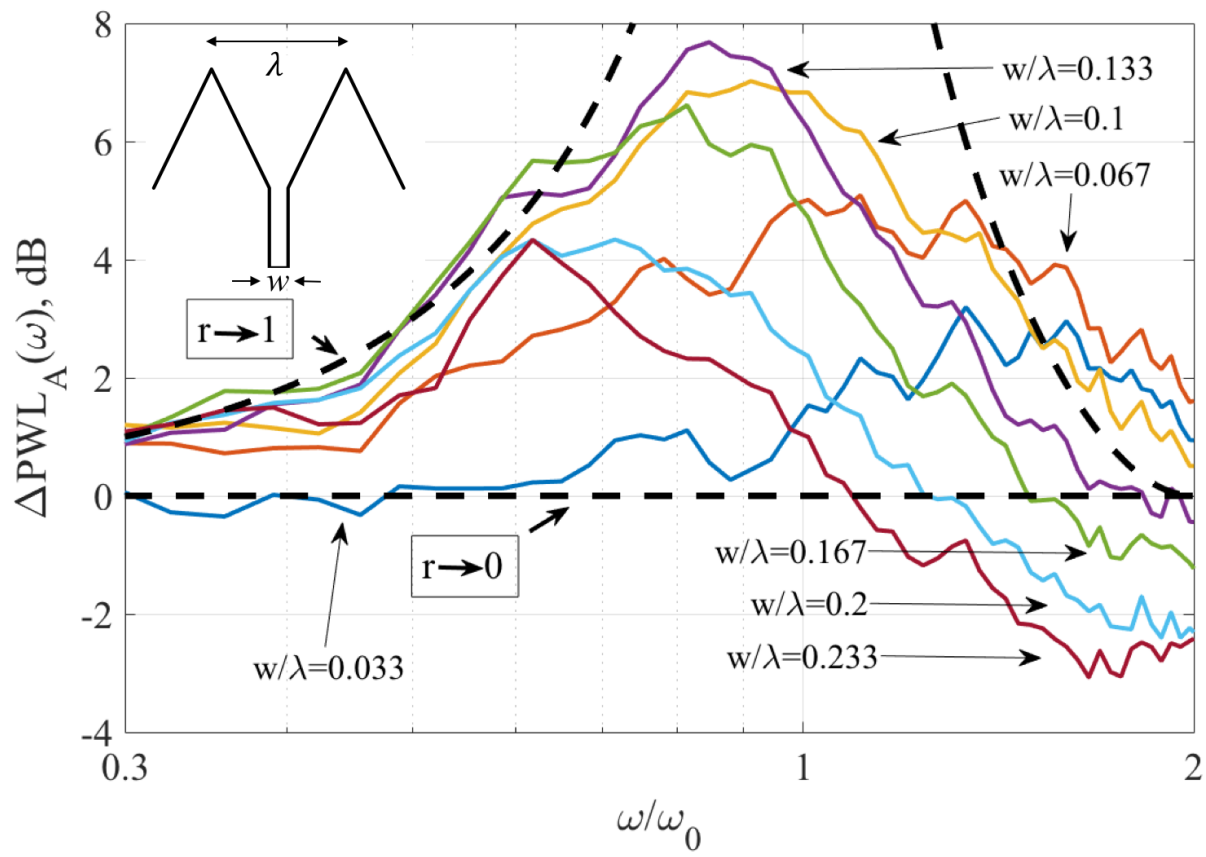

FIG. 7. Additional sound power reduction comparisons for a slitted-root serration for varying slit width $w / \lambda$ at fixed slit amplitude $h_{s} / c_{0}=0.133$, serration amplitude $\left(h / c_{0}=0.167\right)$ at jet velocity of $60 \mathrm{~m} / \mathrm{s}$.

- For all slit widths $w$ investigated, levels of noise reduction always exceed those of the un-slitted sawtooth serrations of the same amplitude. The exception is at high frequencies where its performance is slightly worse compared to the sawtooth profile (by a few $\mathrm{dB}$ ). We emphasize that this geometry still provides noise reductions compared to a straight leading edge.

- The smallest slit width $w / \lambda=0.033(w=0.5 \mathrm{~mm})$ under investigation provides almost identical noise reductions (to within $0.3 \mathrm{~dB}$ ) at frequencies $\omega / \omega_{0}$ below 0.6 . Above this frequency, this slitted geometry shows slightly better noise reductions (up to $2 \mathrm{~dB}$ ) compared to the un-slitted sawtooth serration as frequency is increased. No clear peak in the noise reduction spectrum around $\omega / \omega_{0}=1$ can be observed suggested that interference does not provide significant additional noise reductions in this narrow slit case.

- As the slit width is progressively increased the levels of noise reduction increase, reaching maximum values at $w / \lambda=0.133$. At slit widths greater than this value, levels of additional noise reduction are observed to reduce. This observation implies the exis- 
tence of an optimum slit width whose additional noise reduction spectra match very closely the theoretical curve $20 \log _{10}\left(s(\omega) \sec \left(\pi \omega / 2 \omega_{0}\right)\right)$ predicted based on interference between two sources of identical strength separated by a distance equal to the slit height $h_{s}$. This observation provides support to the noise reduction mechanism proposed in Section II B. As $s(\omega)=1$ is assumed in this prediction, this theoretical curve therefore represents the upper limit on the noise reduction that can be obtained through the use of slitted-root serrations.

- The two theoretical spectra $20 \log _{10} s(\omega)$ and $20 \log _{10}\left(s(\omega) \sec \left(\pi \omega / 2 \omega_{0}\right)\right)$ therefore represent the lower and upper limits on the noise reductions that may be achieved using slitted profiles additional to the noise reduction on un-slitted profiles. All additional noise spectra therefore fall within these two limits.

- The peak frequency $\omega / \omega_{0}$ may be seen to reduce from above 1 to below 1 as the slit width is increased from $w / \lambda=0.033$ to 0.233 . This observation suggests that the effective source separation distance $h_{s}^{*}$ must be different from the physical slit height $h_{s}$. The effective (acoustic) centers of each source is therefore not precisely located at the ends of the slit, which vary with the slit width.

Figure 8 is a plot of the overall sound power reductions compared to a straight edge integrated over the non-dimensional frequency range of $\omega / \omega_{0}$ of between 0.3 and 3 plotted against non-dimensional slit width $w / \lambda$. It provides explicit evidence for the existence of an optimum slit width (of the measured test cases), which in this case is $w / \lambda=0.167$ representing a slit width of just $2.5 \mathrm{~mm}$. However, increasing or reducing this slit width by just $0.5 \mathrm{~mm}$ causes a significant decrease in noise reductions, indicating that the source balance between $q_{r}$ and $q_{w}$ is highly dependent on slit width, as we shall show in section VII below.

\section{INFLUENCE OF $h_{s}$ ON ADDITIONAL NOISE REDUCTIONS}

In this section we investigate the influence of the slit height $h_{s}$ on the noise reductions. The additional noise reduction spectra $\triangle P W L_{A}$ are plotted against non-dimensional frequency $\omega / \omega_{0}$ in Figure 9 for eight different slit heights $h_{s} / 2 h$ of $0.2,0.4,0.6,0.64,0.7,0.8$, 0.9, 1 (obtained by adjusting the inclination angle of the oblique edge). Note that $h_{s} / 2 h=1$ represents the limiting case of only slits without a sawtooth profile, as sketched in Figure 9. 


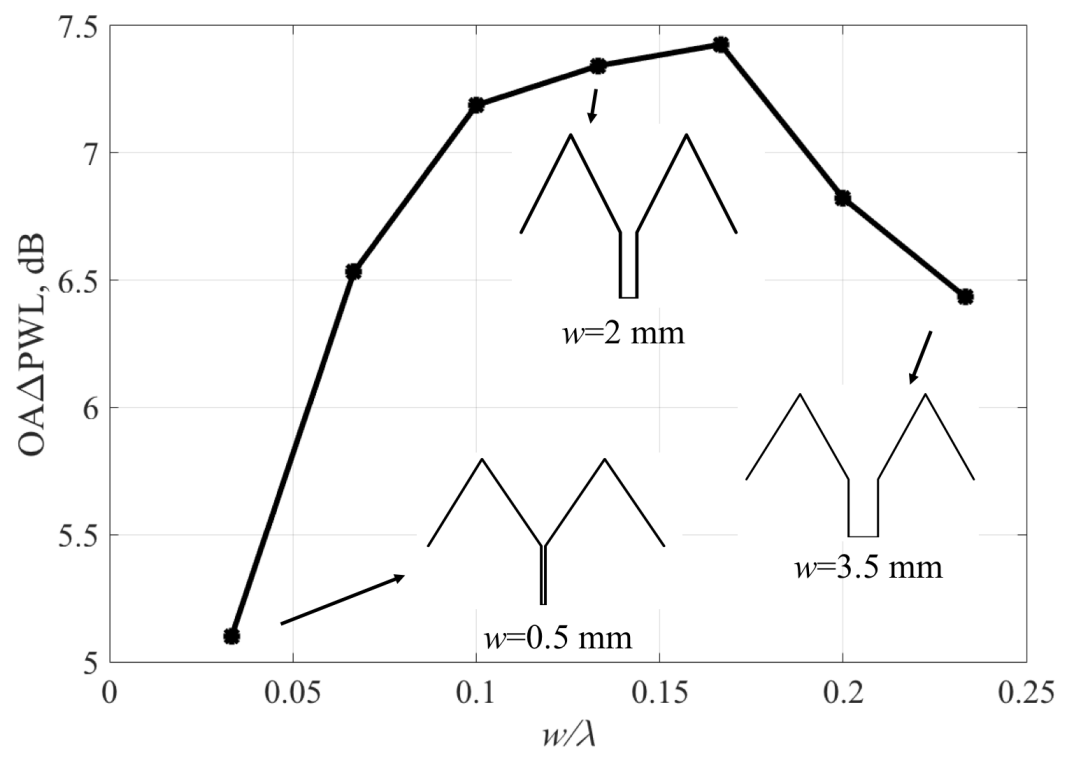

FIG. 8. OAPWL versus $w / \lambda$ for a slitted-root serration for varying slit width $w / \lambda$ at fixed slit amplitude $h_{s} / c_{0}=0.133$, serration amplitude $h / c_{0}=0.1$ at jet velocity of $60 \mathrm{~m} / \mathrm{s}$.

In this figure the overall amplitude $2 h / c_{0}$ is kept constant at 0.33 , the slit width is kept constant at the optimum value (of the measured test cases) identified in figure 7 of $w / \lambda=0.133$ and the serration wavelength kept constant at $\lambda / c_{0}=0.1$ corresponding to four times the turbulence integral length-scale.

The peaks in the noise reduction spectra in Figure 9 of maximum noise reduction all occur close to the tuned frequency of $\omega / \omega_{0} \approx 1$. Note that variations in the peak frequency $\omega / \omega_{0}$ are likely to be for the same reason as for the varying peaks in figure 7 , which can be explained by the varying effective separation distance $h_{s}^{*}$ with slit height $h_{s}$.

A clear trend in the noise reduction spectra is observed. As the slit height $h_{s}$ is increased the peak frequency $\omega / \omega_{0}$ of maximum noise reduction approaches, from below, a value of approximately 1.25 while the level of maximum additional reduction increases, reaching a level of approximately $11 \mathrm{~dB}$ (compared to a conventional sawtooth profile of the same amplitude). Based on the model presented in section II B, this finding suggests that, as $h_{s}$ is decreased, the sources at either ends of the slit become more equal in strength and their effective separation distance approaches a limiting value corresponding to an effective slit height of $0.8 h_{s}$ (corresponding to a peak frequency of $\omega / \omega_{0}=1.25$ ).

To quantify the sensitivity of the noise reduction performance to slit height, the overall sound power reductions were calculated over the non-dimensional frequency range of $f h / U$ 


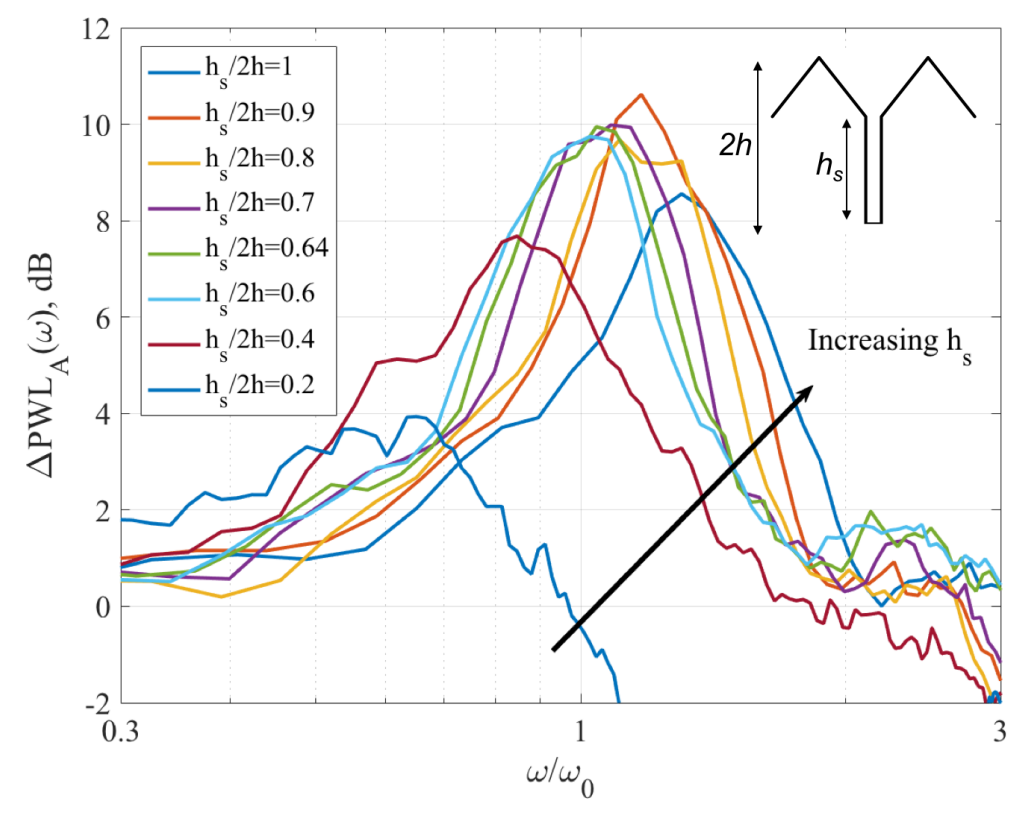

FIG. 9. Additional noise reductions $\triangle P W L_{A}$ versus non-dimensional frequency $\omega / \omega_{0}$ for a serration ampltiude $h / c_{0}=0.167$, wavelength $\lambda / c_{0}=0.1$ and jet velocity $U=60 \mathrm{~m} / \mathrm{s}$.

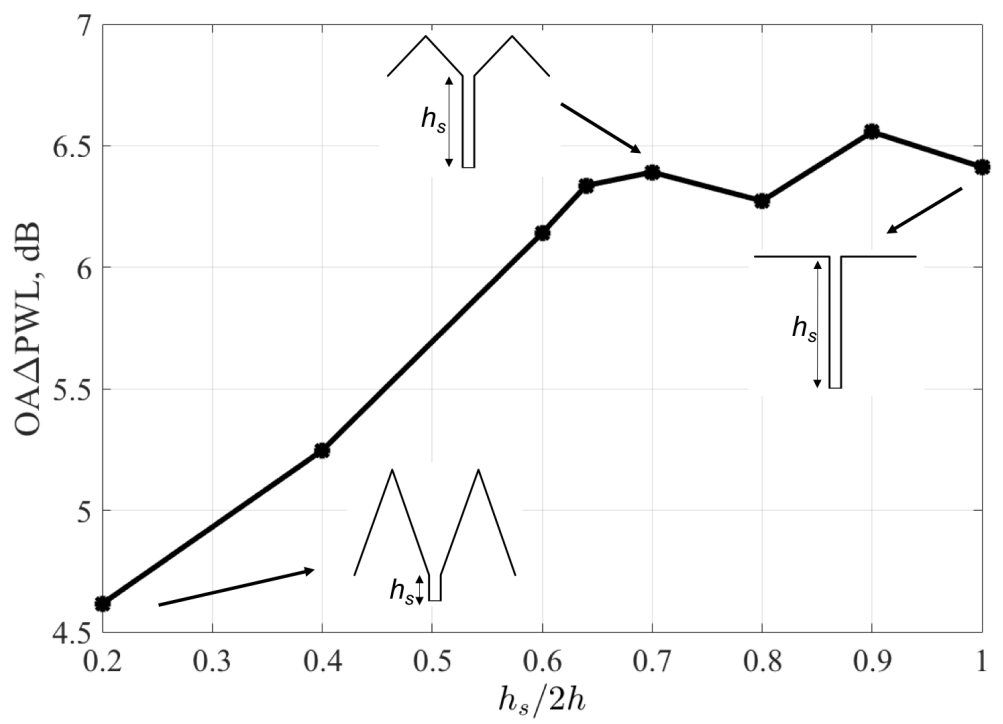

FIG. 10. Overall sound power reductions $O A \Delta P W L$ versus $h_{s} / 2 h$ for a serration amplitude $h / c_{0}=0.167$, wavelength $\lambda / c_{0}=0.1$ and jet velocity $U=60 \mathrm{~m} / \mathrm{s}$.

of between 0.15 and 2 and plotted against $h_{s} / 2 h$ in Figure 10.

For this combination of slit width, wavelength and frequency bandwidth, the overall noise reduction can be observed to increase sharply with increasing slit height up to a value of about $h_{s} / 2 h=0.6$. Above this value, overall noise reductions appear to fluctuate by nearly 
$1 \mathrm{~dB}$ even though the frequency of maximum noise reduction reduces as $1 / h_{s}$, according to Eq. (11). The extreme value $h_{s} / 2 h=1$ in this figure corresponds to the special case in which only slits are present at the leading edge with no sawtooth roots. Simply adding narrow slits (just $2 \mathrm{~mm}$ in this case) is therefore capable of providing considerably greater noise reductions than conventional single-wavelength or sawtooth profiles alone. The effectiveness of introducing slits to a 5\% thick 3D aerofoil typical of an Outlet Guide vanes will be examined in section VIII.

\section{COMPARISON BETWEEN PREDICTED AND MEASURED ADDITIONAL NOISE REDUCTIONS}

To assess the validity of the noise reduction mechanism described by Equation (10) in Section II B for predicting the noise reductions additional to the baseline (un-slitted sawtooth geometry), predictions from this equation are compared to the measured additional noise reduction spectra for the three different slit heights of $h_{s} / 2 h=0.4,0.7$ and 1 . In this comparison the slitted root profile has a peak-to-root distance $2 h / c_{0}=0.33$, a slit width of $w / \lambda=0.133$, and at a flow speed equal to $60 \mathrm{~m} / \mathrm{s}$. In figure 11 the measured noise reduction spectra plotted against $\omega / \omega_{0}$ is compared against the predicted spectra with assumed values of $s(\omega)=1$ for the three values of $r(\omega)$ of $0.4,0.53,0.45$ chosen to provide best fit to the measured data for each value of $h_{s}$ over the frequency range of interest. Note that, for simplicity, we have assumed that $r(\omega)$ is independent of frequency. These simulations also assume a value of $s(\omega)=1$, which we know to be correct only in the low frequency limit. However, its precise value at higher frequencies is unknown and is therefore a source of error in the noise reduction predictions. Finally, the slit height $h_{s}$ in the expression for the tuned frequency $\omega_{0}=\pi U / h_{s}$ is adjusted by no more than $20 \%$ of the physical value to give best fit to the frequency of maximum noise reduction. This slit height $h_{s}$ may therefore be regarded as the effective slit height $h_{s}^{*}$ corresponding to the streamwise separation distance between the two sources at either ends of the slit.

Very good levels of agreement are obtained over the entire frequency range with the measured noise reduction spectra by the simple model obtained by adjusting just the two parameters of $\omega_{0}$ and $r(\omega)$ (where $\omega / \omega_{0} \approx 1$ ), whilst assuming $s(\omega)=1$.

The key to obtaining good levels of noise reduction by the use of these profiles is to 


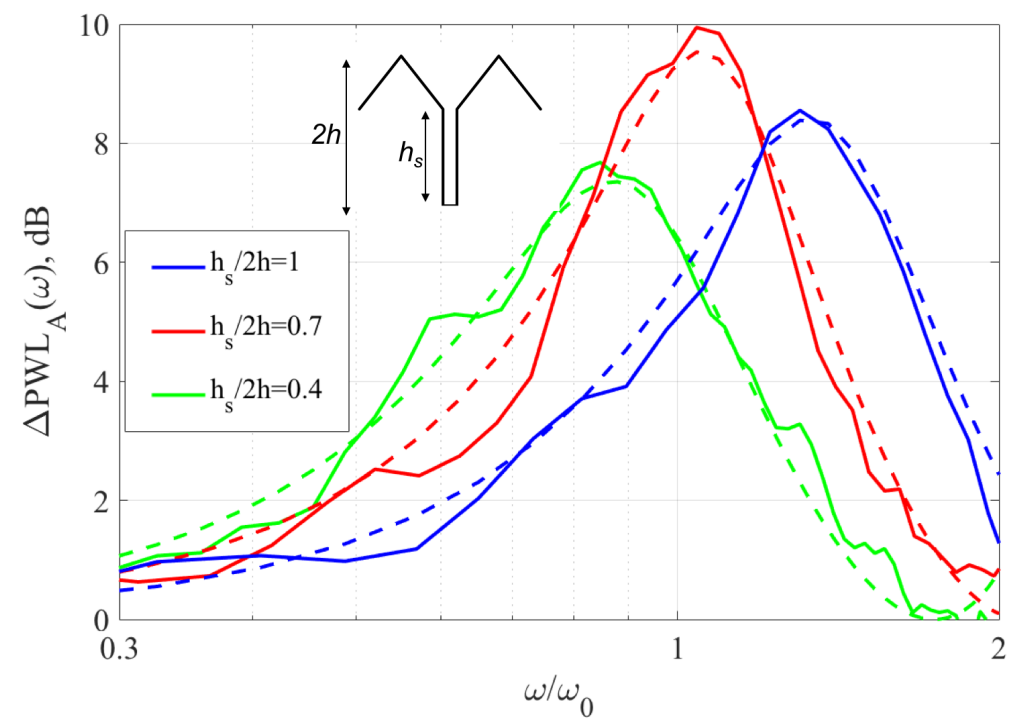

FIG. 11. Comparison between analytic and experimental noise reductions for three different slitted serrations of $h_{s} / 2 h=0.4,0.7$ and $1\left(h_{s}^{*} / h_{s}=1.15,0.95,0.75\right)$, at a fixed $w / \lambda=0.133,2 h / c_{0}=0.33$, $\lambda / c_{0}=0.1$ at jet velocity $\mathrm{U}=60 \mathrm{~m} / \mathrm{s}$.

ensure that the source strengths at either ends of the slit are as equal as possible at the tuned frequency $\omega_{0}$. It is therefore important to understand how the ratio of source strengths $r(\omega)$ varies with both $h_{s}$ and $w$. An estimate for $r(\omega)$ for any slitted-root geometry may be obtained from the value which most closely matches the predicted noise reduction spectra to the measured spectra.

Figure 12 shows the variation of $r(\omega)$ (black line) and $h_{s}^{*}$ (red line) with slit height obtained by following this procedure. This figure suggests that $r(\omega)$ tends from above towards a maximum value of 0.55 as $h_{s}$ increases. Unsurprisingly, the variation of $r$ with $h_{s}$ follows a similar variation to the overall sound power variation plotted in Figure 10, since noise reductions are predicted to increase as $r$ tends to 1 . We emphasize that the estimate of $r(\omega)$ in Figure 12 are only approximate values owing to the erroneous assumption that $s(\omega)=1$ and that $r(\omega)$ is frequency independent. Additional sources of error are also introduced by the assumption that the turbulence is frozen as it convects between the serration peak and the root where in practice some de-correlation of the turbulent flow will occur. Nevertheless, Figure 12 provides useful information about the design of the slitted profiles for maximum noise reduction.

Finally, we consider the variation in source strength ratio $r(\omega)$ with slit width, which we plot in figure 13 against slit width $w / \lambda$ normalized on $\lambda$. The results for 7 values of slit 


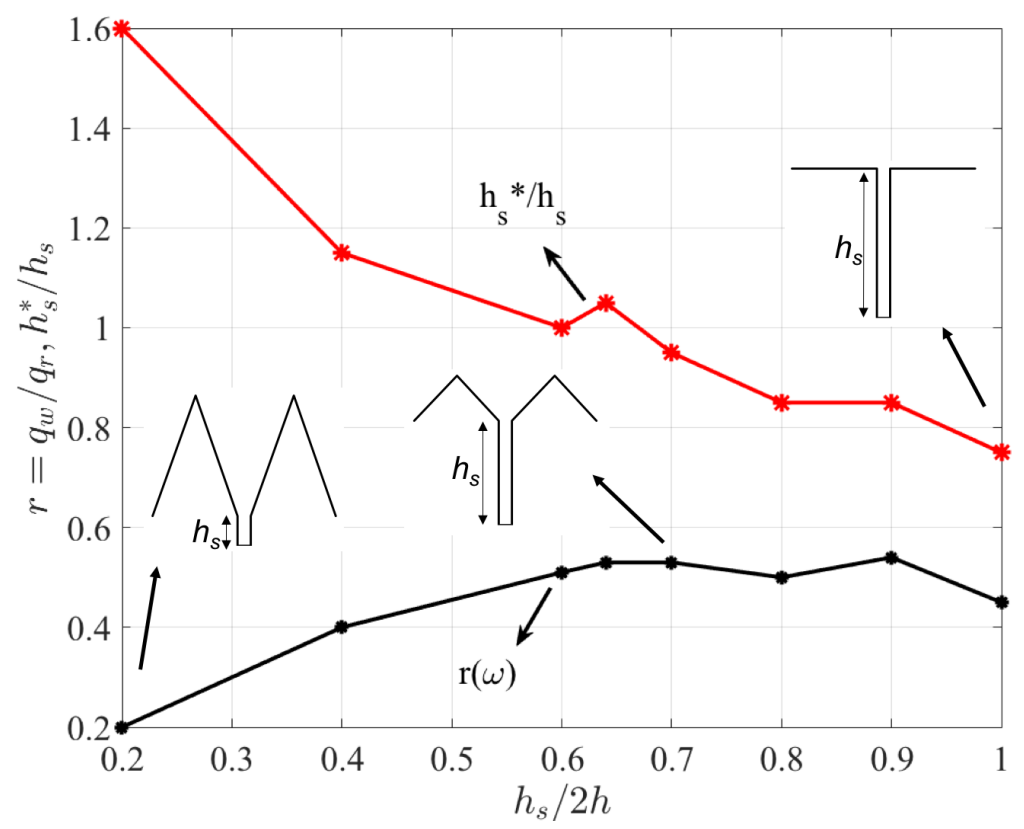

FIG. 12. Source strength $r$ versus $h_{s} / 2 h$ (Black line) and ratio of effective slit height to actual slit height calculated from the measured data (Red line) .

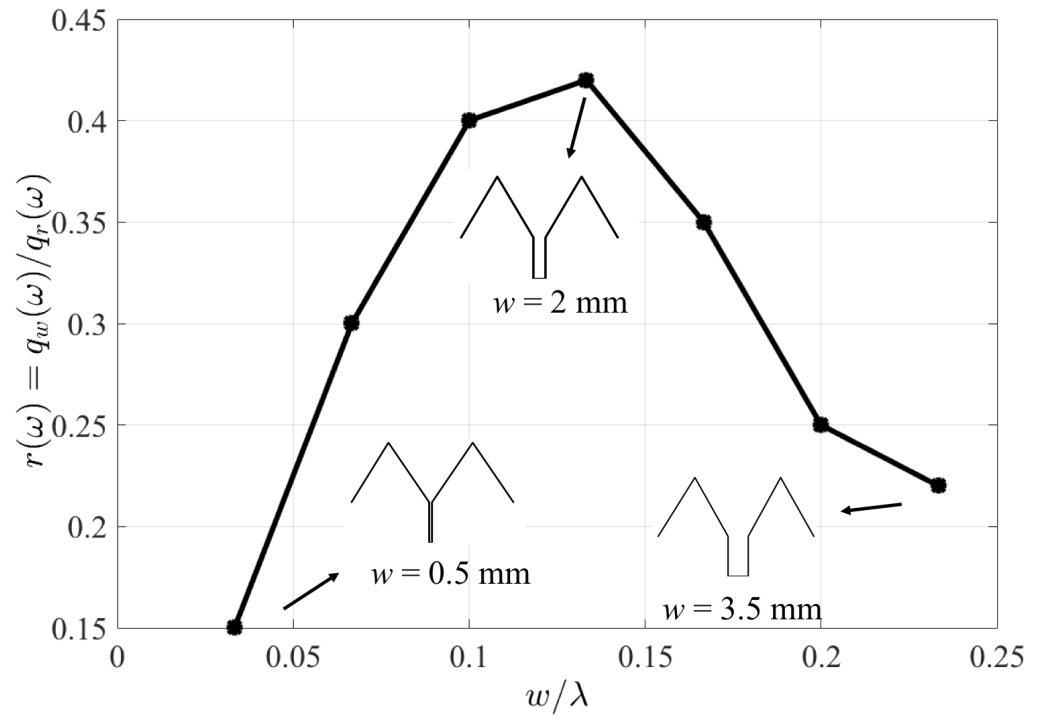

FIG. 13. Source strength $r$ versus $w / \lambda$ calculated from the measured data.

440 width are plotted for $h_{s} / 2 h=0.4$. Unlike with the variation in $h_{s}$, a clear optimum value 441 of $w / \lambda=0.133$ in slit width (corresponding to an actual width of just $2 \mathrm{~mm}$ ) is observed. 


\section{SOUND POWER SPECTRA COMPARISON FOR 3D AEROFOILS}

Previous sections have demonstrated the superior performance of slitted leading edge profiles on reducing interaction noise compared with conventional serrations of the same amplitude on flat plates. In this final section we demonstrate the control principle of destructive interference obtained through the use of slits on the aerofoil leading edge of realistic 3D aerofoils.

A symmetric thin aerofoil of $5 \%$ thickness (Typical of an Outlet Guide Vane) of $0.15 \mathrm{~m}$ chord was investigated in this study. To maximize the noise reduction at low frequencies, only narrow slits are introduced at the leading edge, i.e., with no sawtooth profile. The slit amplitude was chosen to be $h / c_{0}=0.167$, separated along the span (the 'wavelength') by $\lambda / c_{0}=0.1$ corresponding to four times the integral length scale. This distance was identified in [10] as being the distance for which adjacent sources on the leading edge are incoherent and therefore do not interfere. The slit width was chosen to be $w / \lambda=0.133$, which was shown in Section $\mathrm{V}$ to provide optimum noise reductions on a flat plate over the frequency bandwidth of interest. A photograph of the slitted profile on the aerofoil leading edge is shown in Figure 14.

The leading edge profile was designed such that if $y(x)=\mathfrak{F}(x)$ defines the aerofoil profile, where $x=0$ represents the trailing edge and $x=1$ the leading edge. The profile $y(X, r)$ at any spanwise position $r$ along the aerofoil is given by,

$$
y(x, r)= \begin{cases}\mathfrak{F}\left(x / c_{0}\right), & 0<x / c_{0}<2 / 3 \\ \mathfrak{F}(x / c(r)), & 2 / 3<x / c(r)<1\end{cases}
$$

This geometry therefore preserves the typical baseline aerofoil profile at all radial position, where the chord $c(r)=c_{0}+g(r)$ is slitted profile as sketched in Figure 1 and $g(r)$ is the profile described about the mean chord line.

The sound power reduction spectra for the 3D slitted aerofoil is plotted in Figure 14 versus $f(2 h) / U$. Also shown in this figure is the corresponding sound power spectra for the slitted profile on a flat plate.

Levels of noise reduction obtained for the slitted profile on the 3D aerofoil are almost 


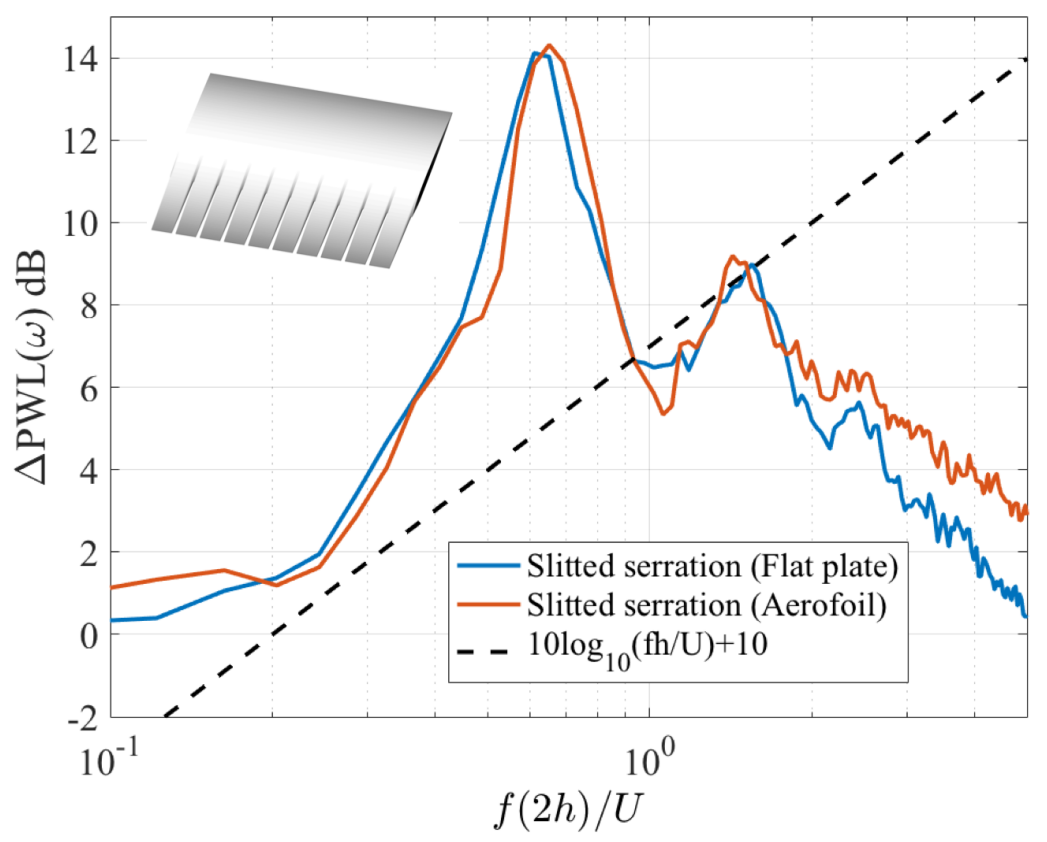

FIG. 14. Sound power reduction comparisons for a slitted serration on flat plate and aerofoil of amplitude $\left(h / c_{0}=0.167\right), w / \lambda=0.133, h_{s} / 2 h=1$ at jet velocity of $60 \mathrm{~m} / \mathrm{s}$.

identical to that for the flat plate at frequencies below about $2 f h / U \approx 2.5(3 \mathrm{kHz})$. Above this frequency, 3D flow effects can be seen to influence the noise reductions by approximately $2 \mathrm{~dB}$ compared to a flat plate. Note that no attempt has been made to optimize this $3 \mathrm{D}$ slitted-root geometry. Additional noise reductions may therefore be obtained, particularly at low frequencies (less than $\omega_{0}$ ) by further tuning of the slit width $w$ and wavelength $\lambda$. The flat plate study presented in section $\mathrm{V}$ therefore provides a good basis for understanding the physics of leading edge serrations and identifying effective leading edge geometries for reducing interaction noise.

\section{CONCLUSION}

This paper has presented a leading profile that is capable of providing considerably better reductions in turbulence-aerofoil interaction noise compared to conventional single wavelength or sawtooth serration profiles. The leading edge profile comprises simply of narrow slits introduced on the airfoil leading edge. By the simple addition of slits, a new control mechanism is introduced involving the destructive interference between two compact source regions approximately located at either ends of the slit. Maximum noise reductions are 
achieved at a frequency, which we refer to in this paper as the tuned frequency at which these two sources radiate $180^{\circ}$ out of phase. Greatest noise reductions are therefore achieved when the two source strengths at either ends of the slit are equal at the tuned frequency. This balancing of source strengths is achieved by the careful choice of slit width. Based on this principle, this paper has demonstrated noise reductions at a flow speed of $40 \mathrm{~m} / \mathrm{s}$ of almost $18 \mathrm{~dB}$ compared with noise reductions of just $7 \mathrm{~dB}$ for a sawtooth un-slitted profile of the same overall amplitude.

A simple analytic model is presented comprising the interference between two perfect coherent compact sources located at either end of the slit. The phase difference between them is governed by the time taken for the turbulent eddys to convect from the front of the slit to the back. By adjusting the relative source strength between these two sources and the total source strength of the slitted-sawtooth compared to the un-slitted profile, good agreement can be obtained with the measured noise reduction spectra.

This paper has demonstrated conclusively that single wavelength or sawtooth leading edge profiles, that have been widely investigated in previous work, provide levels of noise reduction that are significantly inferior to those achievable by the new design. Further work is needed to understand the factors that control the source strength distribution along the edge to optimize the leading edge profile further. Further refinement of the slitted profiles are also possible that may be rounder and avoid sharp corners as long as the source balance is not affected. Finally, further work is needed to quantify the effect of slits on aerodynamic performance. Any degradation in aerodynamic performance must of course be balanced against the positive effects of reduced noise.

\section{ACKNOWLEDGMENTS}

This work was partly supported by the EPSRC (EP/J007633/1) and by InnovateUK, HARMONY Programme $\left(G A n^{\circ} 101367\right)$. Rolls-Royce Plc is also acknowledged for the financial and technical support given. 
[1] P.T. Soderman, "Aerodynamic effects of leading-edge serrations on a two- dimensional airfoil", NASA TM X-2643 (1972).

[2] A.S. Hersh, P.T. Soderman and R.E. Hayden, , "Investigation of acoustic effects of leading edge serrations on airfoils", in Journal of Aircraft, 11(4), 197-202 (1974).

[3] M. Roger, C. Schram and C. \& L. De Santana, , "Reduction of Airfoil Turbulence-Impingement Noise by Means of Leading-Edge Serrations and/or Porous Materials", in AIAA/CEAS Aeroacoustics Conference, 2013-2108 (2013).

[4] V. Clair, C. Polacsek, T. Le Garrec, G. Reboul, M. Gruber. and P. Joseph, , "Experimental and Numerical Investigation of Turbulence-Airfoil Noise Reduction Using Wavy Edges ", in AIAA Journal, 51(11), 2695-2713 (2013).

[5] Alex S.H. Lau, Sina Haeri and Jae Wook Kim, , "The effect of wavy leading edges on aerofoilgust interaction noise ", in Journal of Sound and Vibration, 332, 6234-6253 (2013).

[6] S. Narayanan, P. Chaitanya, S. Haeri, P. Joseph, J. W. Kim and C. Polacsek, , "Airfoil noise reductions through leading edge serrations ", in Physics of Fluids, 27, 025109 (2015).

[7] T. P. Chong, A. Vathylakis, A. McEwen, F. Kemsley, C. Muhammad and S. Siddiqi, , "Aeroacoustic and Aerodynamics performance of an aerofoil subjected to sinusoidal leading edges", in 21st AIAA/CEAS Aeroacoustics Conference, AIAA 2015-2200 (2015).

[8] B. Lyu, M. Azarpeyvand and S. Sinayoko, , "Noise prediction for serrated leading-edges ", in 22nd AIAA/CEAS Aeroacoustics Conference, AIAA 2016-2740 (2016).

[9] J.W. Kim, S. Haeri and P. Joseph, , "On the reduction of aerofoil-turbulence interaction noise associated with wavy leading edges ", in Journal of Fluid Mechanics, 792, 526-552 (2016).

[10] P. Chaitanya, P. Joseph, S. Narayanan, C. Vanderwel, J. Turner, J. W. Kim and B. Ganapathisubramani, , "Performance and mechanism of sinusoidal leading edge serrations for the reduction of turbulence-aerofoil interaction noise" , in Journal of Fluid Mechanics, 818, 435-464 (2017).

[11] Fernando Gea-Aguilera, James R. Gill, David Angland and Xin Zhang, , "Wavy Leading Edge Airfoils Interacting with Anisotropic Turbulence Read More", in 23rd AIAA/CEAS Aeroacoustics Conference, AIAA 2017-3370 (2017).

[12] P. Chaitanya, S. Narayanan, P. Joseph and J. W. Kim, , "Leading edge serration geometries 
for significantly enhanced leading edge noise reductions", in 22nd AIAA/CEAS Aeroacoustics Conference, AIAA 2016-2736 (2016).

[13] Jacob M. Turner and Jae Wook Kim, , "Aeroacoustic source mechanisms of a wavy leading edge undergoing vortical disturbances ", in Journal of Fluid Mechanics, 811, 582-611 (2017).

[14] T. P. Chong, P.F. Joseph and P. O. A. L. Davies, , "A parametric study of passive flow control for a short, high area ratio $90^{\circ}$ curved diffuser ", in Journal of Fluids Engineering, 130(11), 111104-12 (2008). 